\title{
Le dire grivois dans un corpus de contes facétieux roumains
}

Micheline Lebarbier

\section{(2) OpenEdition}

1 Journals

Édition électronique

URL : https://journals.openedition.org/clo/1506

DOI : $10.4000 /$ clo. 1506

ISSN : 2266-1816

Éditeur

INALCO

\section{Édition imprimée}

Date de publication : 1 janvier 2012

Pagination : 141-167

ISBN : 978-2-85831-206-1

ISSN : 0396-891X

\section{Référence électronique}

Micheline Lebarbier, "Le dire grivois dans un corpus de contes facétieux roumains », Cahiers de littérature orale [En ligne], 71 | 2012, mis en ligne le 27 avril 2014, consulté le 01 juillet 2021. URL: http://journals.openedition.org/clo/1506 ; DOI : https://doi.org/10.4000/clo.1506

Ce document a été généré automatiquement le 1 juillet 2021.

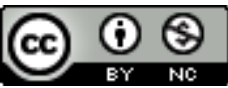

Cahiers de littérature orale est mis à disposition selon les termes de la Licence Creative Commons Attribution - Pas d'Utilisation Commerciale 4.0 International. 


\title{
Le dire grivois dans un corpus de contes facétieux roumains
}

\author{
Micheline Lebarbier
}

1 Lors de leur recueil, au cours de l'automne-hiver 1971-1972 dans trois villages de la Roumanie communiste, les contes facétieux étaient considérés par les habitants comme « des blagues, des mensonges, des bêtises ». Ils se racontaient le soir à la veillée, l'hiver surtout, lorsque m'a-t-on dit on s'ennuie. On racontait en famille aux enfants, ou on se rassemblait chez l'un ou chez l'autre pour effectuer un travail en commun, ou pour le plaisir d'être ensemble. Au cours de ces soirées, si on racontait surtout aux enfants pendant les veillées familiales des histoires merveilleuses peuplées de dragons, de nobles chevaliers et de princesses, on racontait aussi, plutôt entre adultes, des histoires plus courtes, des histoires incisives qui étaient un reflet caricaturé des relations humaines : entre maître et serviteur, entre époux, ou encore avec l'Autre, l'étranger, celui qui reste, qui doit rester à la marge de la société, et qui s'avéra être souvent le Tsigane. Dans le nord, dans le Maramureş, des veillées appelées « veillées de filles pour faire venir les garçons " étaient organisées pendant l'hiver pour permettre les rencontres et favoriser les mariages. Divers jeux et distractions y étaient pratiqués et les contes facétieux y avaient leur place.

2 On ne racontait pas qu'aux veillées, on racontait aussi aux champs lors des pauses, aux mariages, à l'armée, à l'hôpital. On racontait pour se distraire et passer agréablement le temps, qu'il soit temps de distraction ou temps de travail. Celui, celle, qui avait un vaste répertoire était considéré(e) comme " celui (celle) qui sait». Cette personne savait des contes, elle était en mesure de captiver son auditoire, de le distraire, de l'amuser. Or comment ces contes amusaient-ils, et aux dépens de qui? Ils amusaient par la caricature et la description de scènes outrancières et la démesure des situations. Les protagonistes de ces scènes allaient du notable qui avait une position élevée dans la hiérarchie sociale - particulièrement s'il incarnait la morale religieuse -, au marginal, trublion de l'ordre établi. Sans oublier celle qui se doit d'être toujours irréprochable, et sur laquelle reposaient (repose encore) l'ordre et l'économie domestiques. L'image caricaturée, en l'occurrence du pope, du Tsigane et de la femme passe, dans ces récits, 
par la transgression de la norme sociale et par le ridicule, châtiment le plus cuisant ${ }^{1}$ de la transgression. Or le ridicule, que le récit se plait à décrire, est tantôt lié à la sexualité lorsqu'elle concerne les débordements du représentant de la morale, tantôt à la scatologie qui concerne celui ou celle qui s'est avili par son comportement déviant ou par sa sottise.

\section{Le corpus}

3 Le corpus, recueilli dans trois villages, regroupe quatre-vingt-dix-sept contes facétieux. Deux de ces villages étaient collectivisés : l'un, Siliştea Snagov, situé à une cinquantaine de kilomètres de Bucarest, l'autre, VădăstriŢa, en Olténie non loin de la frontière bulgare. Breb, le seul à ne pas avoir été collectivisé se trouve dans le Maramureş, au nord de la Roumanie à environ vingt-cinq kilomètres de la frontière, alors soviétique. C'était le village dont le mode de vie était resté le plus traditionnel, et celui où la collecte des contes a été la plus fructueuse. Les occasions de conter (veillées de travail collectif ou de distraction, ou encore veillées familiales) étant plus nombreuses, les récits circulaient d'autant mieux.

Le recueil des contes s'effectua auprès de narrateurs et narratrices adultes, parfois auprès d'adolescents ou d'enfants dans les villages d'olténie et du Maramureş. Ces contes étaient considérés par les villageois comme des «blagues, des bêtises » qui ne se disaient que dans un certain contexte. Il faut signaler que plusieurs enregistrements ont été suscités, les conditions de l'enquête n'étaient pas toujours réunies lors de la collecte. Lorsqu'il est arrivé que j'assiste à une veillée de travail (entre femmes), elles me prièrent de laisser le magnétophone dans la pièce d'à côté pour pouvoir se laisser aller à dire ces fameuses "bêtises ». Le lendemain, lorsque j'ai sollicité l'une ou l'autre des participantes pour enregistrer les récits entendus la veille, la présence du magnétophone, le contexte différent en dehors de l'émulation collective, entraînèrent des énoncés, une prosodie quelque peu modifiés. D'autre part, il s'avéra que les femmes racontaient plus volontiers entre elles (« entre-nous entre filles») des récits à contenu sexuel. Les hommes en racontaient aussi entre eux et les enfants avaient un répertoire qui leur était propre et qu'ils ne racontaient pas devant les adultes. Deux textes recueillis auprès d'un adolescent concernent l'un et l'autre la découverte de l'anatomie féminine et l'apprentissage de la sexualité. Aussi, est-ce probablement dû aux conditions de l'enquête et à ma présence -féminine et étrangère, le corpus ne recèle-til que quelques récits ayant trait à la sexualité et accessoirement à la scatologie; ou bien il s'agit d'un motif' survenant au cours de la narration.

\section{La place de ces motifs dans le corpus}

Sur quatre-vingt-dix-sept contes recueillis :

- six récits parlent sans détour des organes génitaux masculins ou féminins ;

- neuf parlent sans détour des relations sexuelles ;

- onze évoquent métaphoriquement des relations sexuelles, lorsqu'il s'agit de l'adultère... féminin exclusivement ;

- neuf lient le pope à la sexualité ;

- sept comportent des motifs scatologiques ;

- cinq mettent en scène la miction, spécialement féminine ; 
- quatre lient le pope à la scatologie.

Ainsi plus de la moitié des récits du corpus comporte un ou plusieurs de ces motifs qui peuvent coexister dans le même conte. Par exemple, le pope peut être lié à des motifs sexuels et scatologiques ${ }^{3}$, quant à la femme... l'énumération ci-dessus le laisse clairement entendre - particulièrement en ce qui concerne les quatre textes où il est question de miction féminine et de sexualité. Cependant, seuls deux contes de ce corpus peuvent être considérés comme totalement licencieux. En ce sens qu'ils recèlent tous les motifs énumérés ci-dessus, sauf les images métaphoriques des relations sexuelles ${ }^{4}$ puisque celles-ci sont nommées et qu'il ne s'agit pas véritablement, en l'occurrence, d'adultère.

7 Ces deux récits ont été recueillis, auprès de Gheorghe, un narrateur de cinquante-neuf ans, à Siliştea Snagov, village situé près du lac Snagov à une cinquantaine de kilomètres de Bucarest. Pour pouvoir enregistrer l'un de ces textes et éviter la gêne des mots crus et des situations scabreuses qui y étaient dépeintes, je dus sortir ainsi que son épouse et laisser le narrateur devant le magnétophone, en présence d'un auditoire exclusivement masculin et du collègue de l'Institut d'Ethnographie et de Folklore de Bucarest chargé de m'accompagner dans mes enquêtes. Il ne m'a pas été possible d'interroger Gheorghe à la suite de l'enregistrement, comme j'ai pu le faire auprès de certaines narratrices avec lesquelles des liens plus étroits avaient été tissés, et qui racontaient d'autres variantes de contes déjà entendus et enregistrés.

Ces deux textes ont déjà été étudiés ailleurs (Lebarbier, 1998), sous l'angle de leur aspect comique. Ici je m'efforcerai d'aborder l'aspect démesuré et transgressif de cette parole ainsi que l'implication personnelle du narrateur... face aux personnes présentes, puis au magnétophone, et à l'auditoire masculin resté pendant l'enregistrement du deuxième récit. Je m'attarderai sur son adhésion plus ou moins importante à cette parole considérée comme " périlleuse » et qui soulève un certain nombre de questions. Questions qui peuvent être reprises à propos de chaque narrateur ou chaque narratrice qui ont raconté des contes où ces motifs ont été abordés.

9 Tout d'abord le contexte du contage : quel contexte suscite plus facilement l'apparition de ces motifs, et leur expression plus ou moins grivoise, ou plus ou moins détournée? Lorsque l'auditoire est exclusivement masculin (veillée aux champs, à la bergerie, à la taverne) ou exclusivement féminin (veillée de travail, filage), ou encore mixte (veillée de travail, veillée de jeunes gens, noces)?

10 Le narrateur est-il dans le rôle que l'auditoire (et la communauté ?) attend de lui lorsqu'il aborde sans détour des sujets ayant trait à la sexualité ou à la scatologie ? Ces sujets sont considérés comme tabous dans toutes les sociétés. Se perçoit-il (elle) comme déprécié(e) ou au contraire valorisé(e) ? Sans oublier de tenir compte de la personnalité propre de chaque individu. Partager un récit (le dire ou l'entendre) sur ces thèmes entraîne-t-il un sentiment de partage, de complicité ou au contraire de gêne entre l'émetteur de ces récits et les récepteurs?

11 Aussi j'aborderai, d'une part l'étude de ces motifs, et d'autre part la rhétorique et la prosodie du narrateur, de la narratrice - et les réactions de l'auditoire, quand la situation le permettait. 


\section{Les motifs sexuels}

Les récits les plus nombreux concernent la femme ${ }^{5}$. Quinze contes mettent en scène l'épouse adultère, profitant de l'absence de son mari pour se livrer à la débauche la plus éhontée; ou bravant tous les interdits, elle sera l'instigatrice de stratagèmes pour outrageusement tromper son mari. Enfin, elle abuse un mari assez stupide pour avoir, à son nez et à sa barbe, des relations avec son amant.

\section{Les mots pour le suggérer... ou le dire}

Dans tous ces textes, sauf devant le mari abusé où l'acte sexuel est décrit comme tel, l'adultère est représenté par une métaphore ( $c f$. note 4). Le plus souvent ce sera le festin que l'épouse s'ingéniera à préparer pour son amant, où le vin coulera à flots et où les mets abondants et rares seront ceux qui règnent sur les tables de fêtes. Le détournement de ces nourritures, fruits du travail (de la femme surtout) et propriétés du foyer, donnera la mesure de l'impardonnable trahison de l'épouse, qui s'avèrera là être autant sexuelle que matérielle. L'excès de la débauche extra conjugale (mais intramuros) sera exprimé par l'ivresse des deux coupables, leurs chants de défi envers le mari et leurs danses endiablées symbolisant une autre forme d'ivresse, celle d'une sexualité débridée. Le mari remettra bon ordre à tous ces excès par une correction bien sentie.

\section{Le mari benêt}

En revanche, dans les deux contes où le mari, soupçonneux, mais benêt, consent à ce que son épouse, qui feint d'être malade, soit «soignée " par son amant, les relations sexuelles sont explicitement décrites et implicitement consenties par l'époux. C'est notamment le cas dans une variante ${ }^{6}$ où l'amant vient vendre des prunes au village, seul remède capable de guérir l'épouse soi-disant malade. L'amant accepte de donner des prunes à la condition que la femme « lui serve... ». Le mari, devant le lit qui tremble, s'effraie de la brutalité de l'acte : «Plus doucement, tu vas tuer ma femme ! - Du diable qu'elle sait mieux que toi le prix des prunes!» répond l'amant; chute du récit sur laquelle se focalise l'aspect comique.

Dans l'autre texte, recueilli à Breb auprès de Ion, l'amant est le docteur. Une femme qui va très (trop) souvent chez le docteur attise les commérages du village et les soupçons de son mari. Les amants parient de coucher ensemble devant lui sans qu'il ne fasse rien. La femme feint d'être malade et tient absolument à ce que son mari l'accompagne chez le médecin pour ne pas être encore accusée.

Ils partirent chez le docteur, elle se mit sur un lit. Il la mit sur le dos et il l'examina pour voir quelle maladie elle avait.

- Bien, fait-il, tu n'as pas une maladie bien grave, fait-il, tu as une petite blessure au... ventre (comme on dit chez nous), mais ce n'est pas une maladie grave. Il fait, on va se débrouiller. Il fait, prends un peu d'onguent, mais seul ton mari peut te faire ce traitement.

- Mais comment?

- Comme ça, fait-il, une autre personne, ce n'est pas permis, seulement le mari.

Le mari regarde et il fait :

- Moi, Monsieur le Docteur? Moi, je ne sais pas, comment je le saurais ? Si tu ne me 
montres pas avant!

- Comment est-ce que je te montrerais, moi ? Il fait, ce n'est pas possible. Enfin, bon!

Le docteur s'écarte et le regarde de nouveau. Il lui montre l'onguent. Il fait :

- Voilà tout le traitement et après ta femme sera bien portante. Mais fait-il, il faut

lui faire.

L'homme prit cinq cents lei de sa poche et les mit dans la poche du docteur. Il fait :

- Seulement, faites-le la première fois et ensuite je le ferai moi.

- Enfin, bon, fait-il, va regarder de la porte.

L'homme regarda. Le docteur alla près de la femme dans le lit, et lui montra comment faire... Il la chevaucha et lui montra comment mettre le médicament. Alors l'homme, de la porte, il se mit à rire, il fait :

- Hé, Monsieur le Docteur, un homme plus bête que moi, fait-il, maintenant il dirait que tu me l'as baisée! simulant la maladie. C'est le stratagème le plus largement utilisé dans ces contes par toutes les épouses, infidèles, paresseuses, voire capricieuses, pour obtenir ce qu'elles veulent de leur mari. Élément qui laisse à penser que seule la maladie feinte ou réelle peut soustraire l'épouse à ses devoirs et à ses tâches et que c'est un des maux les plus redoutés par le mari pour qu'il consente à accéder aux demandes de sa femme.

En revanche, soulignons que le sexe féminin est, de façon euphémique, dénommé ic «le ventre » et que Ion installe une distance entre son énoncé et lui, puisqu'il le fait prendre en charge par la communauté : «Comme on dit chez-nous». Alors qu'un terme cru existe pour désigner le sexe féminin. Il est d'ailleurs couramment employé dans une insulte que l'on traduirait en français par «Va te faire voir!» Du-te în pizda mă-ti littéralement « Retourne dans la chatte de ta mère », et qui est plus pudiquement repris par Du-te în mă-ta "Retourne dans ta mère ", éludant ainsi le terme pizda «chatte, con, au sens de vagin». On retrouvera ce terme dans les deux récits de Gheorghe, mentionnés plus haut, où les organes sexuels féminins et masculins sont nommés sans euphémismes. On y reviendra. En revanche, l'acte sexuel est ici désigné dans son acception la plus vulgaire : mi-ai regulat-o «tu $\boldsymbol{m e}$ l'as baisée $»^{7} ; \boldsymbol{m i -}$ le datif du pronom personnel à la première personne indiquant bien que c'est au mari que l'acte s'adresse en définitive... s'il acceptait de le comprendre.

Quant à l'onguent, il symboliserait le sperme que seul le mari peut mettre «dans le ventre " de sa femme. Si l'on peut y voir un stratagème pseudo médical, le médecin étant le seul à connaître l'usage des onguents et autres pommades et le seul à pouvoir éduquer la population à leur emploi, on pourrait y voir aussi le déniaisement ${ }^{8} \mathrm{~d}^{\prime} u n$ mari benêt, certes, et peut-être aussi peu au fait des pratiques sexuelles. L'amant vendeur de prunes réplique, lui, au mari, que son épouse connaît mieux que lui la valeur des prunes. Le mari, n'en aurait-il donc aucune idée ? Aucune idée du plaisir féminin?

Pour l'initiation à la reproduction, l'observation des animaux était évidente, mais pour l'initiation à la sexualité peut-être n'était-elle pas suffisante. Cette société traditionnelle, où lors de l'enquête n'existait pas encore la télévision et à peine la radio, offrait peu d'occasions pour les adolescents de découvrir la réalité des relations conjugales. Certes, il existait les veillées de jeunes gens où des récits, des chants et des jeux à connotation érotique étaient pratiqués, mais ils concernaient une tranche d'âge plus avancé, celle des jeunes en âge de se marier et on peut se demander si le mari benêt avait participé à ces veillées ou encore s'il avait compris le sens de certains jeux. Deux récits recueillis à Breb auprès de Petru, un adolescent de quatorze ans, laissent 
transparaître cette ignorance et cette préoccupation. Les deux textes décrivent les tribulations du garçon ignorant et leurs titres sont explicites: "Un garçon qui cherchait à se marier» et "Un garçon qui est allé l'apprendre" dépeignent les tentatives malheureuses de chaque protagoniste pour découvrir l'anatomie féminine et la sexualité. On retrouve la notion d'apprentissage pour lequel il faut payer (cinq cents lei là aussi, dans l'un des textes), mais où la naïveté du garçon est abusée par la perfidie de la supposée initiatrice. Elle lui fait croire soit que boire du thé équivaut à faire l'amour, évoquant ici une blague de Radio Erevan (cette radio était à l'époque très écoutée et ses blagues souvent politiquement subversives circulaient au cours de rassemblements les plus divers), soit que le sexe féminin mord, évoquant là le mythe du vagin denté, constante de l'imaginaire masculin'. Outre le risque « pour les petits mâles de devenir... nourriture » (Lascault, 2008, 50), ceux-ci redouteraient l'inconnu qu'est pour eux le sexe féminin. Cet inconnu est d'autant plus présent dans le récit qu'il est raconté par un adolescent. Le récit se termine par l'étonnement du garçon qui, lors de la nuit de noces, découvre que le sexe de sa femme, lui, «ne mord pas ». Cependant, une certaine connaissance serait nécessaire, car le garçon du premier conte est abandonné le soir de ses noces par la mariée effarée devant son ignorance ${ }^{10}$. Cela ne va pas sans rappeler un des contes du Cycle de Păcală (le héros facétieux de la littérature orale roumaine ${ }^{11}$ ) où la mariée s'enfuit avec lui et plante là un imbécile de mari (Lebarbier, 2011, 78 et ss). Ces récits éclairent sur la crédulité du mari benêt, mystifié par sa femme adultère et son amant, docteur ou marchand de prunes.

Quoi qu'il en soit, qu'il soit dupé ou qu'il rétablisse l'ordre dans son foyer (et son honneur) par une vigoureuse correction, le mari trompé des contes facétieux est le plus souvent considéré implicitement comme un naïf qui se laisse manipuler par sa femme ${ }^{12}$. Par exemple, il part au loin chercher un remède impossible pour une épouse prétendument malade, qui souhaite l'éloigner pour passer du bon temps avec son amant, ou bien il se contente d'un fade brouet alors que des mets raffinés sont préparés (et dissimulés) pour l'amant. Un adjuvant extérieur lui sera, dans la plupart des textes, nécessaire pour que ses yeux soient dessillés et que l'ordre conjugal soit restauré.

\section{L'épouse adultère}

21 Dans ces récits de femmes infidèles, les relations sexuelles sont exprimées sous forme de métaphore. Dans un des textes, l'ivresse de l'épouse (du pope en l'occurrence) est mentionnée et son "amant hennit dans la maison comme un étalon». Outre la puissance sexuelle de l'animal associée à l'amant, ses hennissements peuvent être vus comme ceux du plaisir. Et outre le festin déjà mentionné et l'ivresse, on trouvera le décompte de l'argent dans le cas d'une femme vénale; ou encore le bavardage dans le grenier dans le cas d'une épouse dupée par son propre mari : « Il l'attrapa par la main et l'attira vers lui, là dans le foin. Ils restèrent ce qu'ils restèrent à bavarder ». Même les chants de défi des amants décrits dans le but d'exposer le comble de la débauche sont très pudiques dans leur expression. Et on comprend mal les précautions oratoires prises par Ioana, une narratrice de Vădăstrị̧a, pour dire la chanson des amants :

- Je connais une chanson, mais la mienne est plutôt gênante [dit l'adjuvant du mari, lors de la découverte de la scène, le mari étant caché dans un sac] ${ }^{13}$.

(Tiens comme j'en dis une maintenant, car la mienne est plutôt gênante) [dit en aparté Ioana]

«Allez, Vladu, sors du sac 
et attrape un gourdin

pour en battre les copains ».

(Lebarbier, 2011, 109)

Ces chants appelés strigături et que je traduis par " chants scandés » se retrouvent dans ce type de conte, répertorié chez Aarne Thompson sous le C.T.1360 C. Ce sont des phrases scandées souvent rimées que le musicien crie sur le rythme de sa musique. Elles comportent souvent des allusions satiriques, et représentent ici l'apogée de l'adultère.

3 Il en va de même des danses endiablées des coupables, généralement associées aux chants scandés : « ... ils commencent tous les deux à faire une danse en se tenant par la taille... La femme scande... » dans un autre chant de défi envers son mari où elle l'enjoint de les tuer. Outre la métaphore sexuelle que représente ici cette danse, dans la vie rurale traditionnelle, la danse a longtemps été une activité institutionnalisée. Jusque dans les années 2000, particulièrement dans le Maramureş, les jeunes gens se retrouvaient avec des musiciens chaque dimanche pour danser sur la place du village ou dans un lieu couvert. Comme les «veillées de filles pour faire venir les garçons », ces rencontres permettaient de nouer des liens devant conduire à des mariages. Or, si les jeunes filles pouvaient danser tout à loisir, il était mal vu qu'une femme danse à partir du moment où elle était mariée. L'épouse qui danse chez elle avec son amant représente bien un flagrant délit d'adultère.

Cependant, dans certains textes qui ne peuvent être considérés comme représentant un adultère, on retrouve les verbes transitifs a iubi pris dans le sens de «faire l'amour », $a$ regula pris dans le sens de «baiser ». Il s'agit de femmes adultères malgré elles, assez naïves pour être bernées par un homme mal intentionné.

\section{L'épouse adultère malgré elle}

Cet homme perfide est souvent le pope toujours prompt, dans ces textes, à convoiter l'épouse d'autrui ou un mari furieux contre le pope qui a profité de la naïveté de sa femme et qui lui rend la monnaie de sa pièce ; comme dans le conte suivant raconté par Ion, le narrateur de Breb. Un jeune marié part à l'armée laissant sa femme enceinte au village. Elle va se confesser ${ }^{14}$ et le pope, attiré par son joli minois, lui fait croire que son mari a laissé en elle un enfant inachevé.

Le premier soir, le pope est venu et il a mis les pieds, le deuxième soir, il vint mettre les mains, le troisième soir, il alla mettre les yeux. Tout jusqu'à ce qu'il soit terminé. Après cela, la femme mit au monde un garçon [...] Quand le mari vint, il alla... directement au berceau voir ce qu'il avait. Il vit comme il était beau ! Ah, quel beau garçon il avait! Alors la femme cria [...] :

- Oui, mais s'il n'y avait pas eu ce pauvre prêtre qui vienne ici, il n'aurait pas de mains, il n'aurait pas de jambes, ni d'yeux, ce ne serait qu'un tronc. Avec ce que tu avais mis, il ne serait pas comme ça.

[...]

Oh, alors le militaire comprit. [...] Le lendemain, il alla chez le pope, car il avait vu que le pope partait de chez lui et lui demanda s'il pouvait lui changer vingt-cinq lei. Il lui dit d'aller chez sa femme, qu'elle avait, elle, de quoi changer. Le militaire alla chez la femme du pope et lui dit que le pope avait ordonné qu'elle lui donne ses deux filles et elle ensuite. D'abord la femme du pope n'a pas voulu, puis elle obéit à l'ordre du pope. Comme ça il lui a baisé les filles d'abord et elle ensuite. 
filles d'abord et elle ensuite ». On retrouve le datif de la troisième personne du pronom personnel [i]-o qui indique que c'est bien vers le père des filles et le mari que l'acte est dirigé, que je traduis par « il lui a baisé... » D'autre part, dans l'acception familière que donne au verbe a regula le dictionnaire DEX, on ne retrouve pas la connotation sexuelle et vulgaire couramment employée en langage parlé. Le DEX lui donne comme deuxième sens (familier) «mettre quelqu'un au pas, lui donner une leçon, le réduire au silence $»^{15}$ (p. 910). Certes, on peut aussi percevoir dans cette expression (que Ion utilise dans ses deux récits) et ses différentes acceptions, la domination de l'homme sur la femme, qui probablement a influé sur l'évolution du sens populaire de ce verbe. Mais on peut également, dans le contexte de ce récit, voir que la leçon a été donnée par le militaire, que le pope et sa famille, humiliés sont contraints au silence, vis-à-vis du village notamment. Rappelons le souci de chacun de ne pas être la cible de moqueries. Le pope est contraint au silence sur son infidélité envers son épouse ${ }^{16}$. La jeune femme et son mari militaire sont contraints au silence sur la naïveté de la jeune épouse enceinte, les filles et la femme du pope qui ont été bernées sont aussi contraintes au silence, puisqu'elles sont l'instrument de la vengeance du militaire. Ce combat entre hommes par l'entremise des femmes se passe dans le secret et en dehors des regards et des commentaires du village. Le pope étant le premier ici, et souvent dans ces textes, à enfreindre la loi qu'il est censé représenter et à en recevoir le «juste» et discret châtiment ${ }^{17}$. Soulignons cependant que le conte facétieux fait la part belle à la crédulité de la femme et à son obéissance aveugle à la parole de son mari, même par personne interposée. Elle permet ainsi à ce combat d'avoir lieu et d'être l'objet de ce règlement de comptes qui va au-delà de sa personne, et de ce qu'elle représente. Si le pope libertin de ce récit est aussi (juste retour des choses) un père et un mari spolié à son tour, il est aussi dans un autre texte, un mari spolié par son facétieux serviteur, Păcală. Il obtient les faveurs de l'épouse du pope par un stratagème identique et par l'obéissance crédule de cette dernière, également identique. Aussi mari infidèle et puni de son méfait par le même type de méfait ${ }^{18}$, ou mari spolié par son serviteur, le pope est la cible favorite des contes facétieux, qui le mettent en scène dans toutes les situations scabreuses possibles, qu'elles soient sexuelles ou scatologiques ou encore sexuelles et scatologiques.

De ce fait, la femme est toujours mêlée à ces deux types de motifs.

\section{Les motifs sexuels combinés aux motifs scatologiques}

Ainsi les deux récits grivois racontés par Gheorghe, et mentionnés plus haut, comportent ces deux types de motifs. On y retrouvera notamment l'épouse du pope bernée par un homme qui se fait passer pour son mari afin d'avoir des relations sexuelles avec elle et se délester d'une infirmité embarrassante. Dans ces deux récits, le narrateur décrit sans métaphores les relations sexuelles - conjugales en l'occurrence ou supposées telles, mais avec ce que je nommerais des « évitements langagiers». Par exemple, on n'y retrouvera pas les termes familiers rencontrés dans les contes étudiés ci-dessus. Mais la miction est le plus souvent désignée sans détour et on verra l'importance qu'elle revêt lorsqu'elle est féminine. Quant aux organes génitaux, dans l'un des textes, ils sont crûment nommés. 
Voici la traduction du texte traitant de gigantisme sexuel tant féminin que masculin. Les termes que j'avais pudiquement traduits dans la publication mentionnée (Lebarbier, 1998, 171) par "vagin » et " pénis » sont exprimés par le narrateur dans la langue populaire : pizda que je traduis ici par «chatte » et pénis pula, que je traduis ici par «bite» (afin d'être au plus près du texte recueilli et du propos du présent numéro $\left.{ }^{19}\right)$. Voici le texte tel qu'il fut raconté par Gheorghe ${ }^{20}$ :

\section{Le gigantisme sexuel}

Un homme avait une fille unique, qui avait une chatte qui s'était tellement développée que sa chatte était encore dans la grange alors qu'elle était dans la maison. Au bout d'un certain temps, elle lui dit :

- Papa, le temps est venu de me marier, va chercher ce qu'il me faut.

- Oh! Quel malheur est sur moi pauvre vieillard!

Il partit. Il passa par un bois... il battait avec sa bite, les glands de la cime du chêne.

- Oh! Mon Dieu, Seigneur ! J'ai trouvé ce qu'il me faut !

- Eh, eh, eh ! dit-il, va plus loin, car je suis le plus petit.

$-\mathrm{Oh}$ ! Encore plus grand ! dit-il.

- Oh, nous savons que c'est pour ta fille, allez va!

Il alla plus loin. Il y en avait un qui rentrait les porcs avec sa bite dans la propriété.

- Oh! dit-il c'est celui-là !

- Non, dit-il, ce n'est pas moi, je suis le plus petit après l'autre. Va au bord de la mer, il fait un pont sur la mer et les gens passent sur sa bite.

Oh là là ! Il arriva là-bas :

- Depuis le temps que je t'attendais, depuis le temps que je t'attendais, oh là là, petit père ${ }^{21}$ ! Bon, va au village chercher neuf roues de charrue, des sacs, et viens qu'on aille à la maison faire la noce avec ta fille.

- Quel péché est sur moi!

Le vieux partit rassembler des roues de par le village là-bas, il mit la bite sur les neuf roues de charrue et même comme ça elle pendait par terre. Allez! En chemin, quand il entra dans la forêt, un loup se mit à manger la bite du garçon. Il dit :

- $\mathrm{Papa}^{22}$, va voir au bout, dit-il, il y a une puce qui me pique.

Le vieux courut, mais il se fatiguait le vieux sur le chemin. Quand il arriva, le loup :

«Ah! Ah! Hé ! » Il revint :

- Qu'est-ce que c'était?

- Un loup la mangeait. C'était ça, dit-il, je te fais un bandage.

Le vieux prit un sac et partit. Il courut là-bas :

- Oh là là ! Que le diable t'emporte ma fille, tu n'es venue que pour m'apporter des ennuis. Il aurait mieux valu que tu meures ${ }^{23}$.

Ils arrivèrent le soir à la maison. Le garçon entra dans la cour et :

- Dressez les tables!

Ils n'avaient pas de chaises. Il dit :

- Laisse, je vais faire la table, les chaises, moi !

Le garçon mit sa bite en guise de chaises autour de la table. La noce arriva, car Untel faisait aussi une noce, le pope vint aussi avec la noce. Comme il était gourmand, le pope mangea et s'enivra, il était au bout, là-bas. En voyant la fille, la bite se dressa et avala le pope. Avalé le pope! Sa femme le cherche, elle le cherche par-ci, par-là (...). Comment trouver le pope ? Il a dû s'enivrer le pope, et aller à la maison. (...) La femme du pope alla à la maison, pas de pope. Le pope se promenait comme chez lui dans la bite du garçon. Il se promenait et le garçon alla... avec la fille. Il coucha avec la fille et rejeta le pope dans la chatte de la fille. La fille dit:

- Reste, dit-elle, il y a une puce qui me pique, je vais pisser.

Elle rejeta le pope plein de... saletés. Il alla à la maison.

- Qu'est-ce qu'il y a, Père ? Il dit :

- Ils m'ont ébouillanté avec de la soupe, femme, et quoi ! Ils ont tous pissé sur moi, 
ils ont jeté toute la soupe sur moi. Ils m'ont ébouillanté. Ils m'ont complètement ébouillanté.

Et elle se mit à laver le pope. sur la description de la démesure des organes de la fille et du gendre. La honte du père revient tout au long de sa quête d'un gendre convenant à sa fille. Il se décrit comme « un pauvre vieillard» accablé du malheur que représente une telle fille. Au-delà de l'éléphantiasis dont elle est affectée et qui accable tant son père, on peut envisager qu'il s'agirait plutôt d'un désordre comportemental, d'un appétit sexuel hors norme - donc honteux, lorsqu'il se lamente sur «le péché qui est sur lui », au point qu'il en vient à regretter que sa fille ne soit pas morte. Malédiction qui représente le comble du reniement pour un parent ${ }^{24}$ et qui semble plus liée à un comportement déviant qu'à une malformation physique ${ }^{25}$. Ce désordre n'apporte que honte et malheur à son père, mais ne semble pas affecter les hommes, on le voit dans la description de la quête du gendre approprié. Le narrateur s'attarde longuement sur les détails qui concernent le pénis du gendre, et les difficultés logistiques quand il s'agit de le transporter et de le soigner ; sa longueur, le nombre de roues nécessaires à son transport, la morsure du loup comparé à une piqûre de puce, le bandage fait avec un sac que l'on suppose être un grand sac, l'essoufflement du père qui court le long de l'organe en question.

D'où les divers usages que l'on peut faire dans ce texte d'un organe masculin démesuré. Notons qu'un euphémisme est aussi utilisé pour nommer le pénis et que l'on retrouve aussi en français : sculă «outil ». Outil aux multiples usages qui, dans ce texte peut servir de gaule, de fourche, de pont, de table, de chaises... Autant de détails descriptifs qui semblent amuser le narrateur et l'auditoire - masculin rappelons-le, sur lequel reposerait le comique du récit, mais qui ne concerne que le pénis. Le vagin, lui, source de tous les tourments, est rapidement décrit dans la première phrase du texte et n'apporte aucun élément comique.

Notons que Gheorghe n'utilise pas les termes qui sont d'usage pour les jeunes mariés, mire "marié », mireasă "mariée», ou bien pour les personnes mariées, bărbat «homme ", femeie «femme », ou encore nevastă «femme mariée ». Il parle des deux protagonistes comme s'ils étaient célibataires, băiatul « le garçon », fata « la fille », alors que l'on assiste à leurs noces.

D'autre part, si la taille des organes des mariés est hors normes, le déroulement du mariage l'est aussi. Lors de l'enquête, et plusieurs textes recueillis en attestent, les mariages se concluaient d'abord entre adultes. Le père ou le parrain du garçon (ou les deux - parfois accompagné de l'intéressé) venaient demander la main de la fille à son père. Cette démarche s'appelait peţit "demande en mariage " et les démarcheurs peŢitori que je traduis par "prétendants ». Or ici c'est le père qui va à contrecœur chercher un mari pour sa fille, et sur l'injonction de cette dernière.

Autre anomalie, le rituel du mariage est escamoté, la noce ne passe pas à l'église, il n'y a pas de cérémonie religieuse, juste les agapes qui concluent chaque noce. Comme si ce mariage entre deux personnes aux caractéristiques sexuelles particulières ne pouvait se faire que de façon païenne, sans même d'engagement officiel entre les deux intéressés, comme si leurs gigantismes respectifs étaient suffisants pour sceller cette union. Mais le pope, s'il ne procède pas au rituel religieux, vient cependant au banquet de noce comme le veut l'usage. Or sa présence n'a de sens que pour le dépeindre dans ses excès, 
leurs conséquences et leur châtiment: il s'enivre et tombe dans le gouffre que représente l'urètre du marié, où il est souillé, sali par les échanges sexuels des jeunes gens. Rejeté à la fois par le sperme de l'un et par l'urine de l'autre, confondus dans la notion de souillure qui affecte le pope et dont sa femme le lave, comme s'il s'agissait d'un nouveau-né qu'on lave des résidus de placenta et de liquide amniotique ${ }^{26}$.

Notons encore que le loup qui mange le pénis du garçon et le pope qui se retrouve dans le vagin de la fille sont l'un et l'autre ressentis comme « des puces qui piquent ». D'une part pour manifester le gigantisme des mariés, mais aussi l'insignifiance et la petitesse (dans tous les sens du terme) du pope. Le pope est mis sur le même plan qu'un loup, animal sauvage et redouté, mais chassé aisément (dans le récit) et d'une puce, minuscule insecte parasite qui pique et dérange, mais dont on se débarrasse aisément, aussi.

On voit par ailleurs que le narrateur utilise sans gêne aucune les termes crus désignant les organes sexuels, l'urine et l'action d'uriner, qui sont nommés sans détour. En revanche, il devient plus hésitant lorsqu'il s'agit de désigner les substances des échanges sexuels. Ils sont mêlés à l'urine et nommés « ... saletés » ou encore assimilés à de la « soupe ». On retrouvera certains de ces évitements dans son autre récit.

\section{Les fesses parlantes}

Il y avait un homme ordinaire, dans un village. Il se maria et une semaine après que son parrain l'ait marié, il devait lui rendre visite avec un cadeau. Le soir, ils se préparèrent avec sa femme, bien comme il faut, avec une gourde, avec tout ce qu'il fallait, une poule rôtie arrangée avec des brioches. Leur chemin passait par un bois. Le garçon marchait dans le bois... sa femme dit :

- Hé, l'homme, dit-elle, je voudrais aller pour... faire ma petite commission.

La femme s'éloigna, et comme sont les femmes d'habitude, elle gratta les feuilles avec un bout de bois. Et en grattant, elle trouva un grain comme celui d'un petit pois. (...) Elle le roule dans sa main, le retourne et hop! elle l'avale. Elle avala le petit pois et son mari s'éloignait. Elle marchait et se rendait compte qu'elle ne le rattraperait pas :

- Hé Ion! mais quelqu'un d'autre répond :

- Hé Ion! plus fort.

- Mais qu'est-ce que c'est que ça, hein ? Mais l'autre :

- Mais qu'est-ce que c'est que ça, hein?

- Oh Seigneur ! mais on entend en bas :

- Oh Seigneur!

- Hé l'homme! Mais :

- Hé l'homme ! plus fort.

[Ils se rendent compte que les fesses de la femme répètent tout ce qu'elle dit. Le mari lui dit de mimer le toast qu'elle doit porter en l'honneur des parrains.]

- Allez, fais comme si tu disais à parrain : "Je vous baise les mains marraine, je vous baise les mains parrain ». Mais :

- Je vous baise les mains marraine, je vous baise les mains parrain.

[Pour éviter que l'on se moque d'eux au village, ils décident de rentrer et d'annoncer qu'elle est devenue muette].

La femme vint tête basse. Quand elle voit son père et sa mère :

- Qu'est-ce que tu as fait ma fille ? Elle...

[Grand malheur dans la famille!]

La femme s'est mise au lit, son père devait aller chercher des sacs vers deux heures $\mathrm{du}$ matin pour aller au moulin. Et l'homme, comme un jeune hein! comment savoir? Il lui est venu l'envie... et il est allé avec sa femme. Entre temps son père l'appelle : 
- Hé Ion!

- Oui ! On entend de là-bas :

- Oui ! Il s'effraie tout seul en voyant ce que c'est. Alors!

- Eh! Dis femme, est-ce que tu parles encore?

- Ben non, je ne parle plus.

- Ah, c'est une maladie avec un remède ça ! C'est une maladie avec un remède, dit-

il. Laisse que ce n'est...

Le pope partait au moulin. Il surveilla le pope jusqu'à ce qu'il sorte. Il s'éloigna un peu et courut.

- Femme.

- Qu'est-ce qu'il y a, Père.

- Ha ! J'ai eu envie de toi et je suis revenu.

- Père, enfin...

- Quoi! Ça suffit!

Il se mêla à la femme du pope, fit ce qu'il fit et partit à la maison.

[La femme du pope s'aperçoit que ses fesses répètent ce qu'elle dit et l'attribue au mauvais œil.]

- Ooh ! Le mauvais œil est venu sur moi. Qu'est que je vais faire ? Quand le pope est venu, c'est là que je l'aurai pris. Ooh ! L'autre :

- Ooh! Le pope est venu sur moi. Eeh! le mauvais œil!

La femme du pope entra dans la maison et ne dit plus un mot jusqu'à ce que le pope arrive.

- Femme! La femme du pope, muette.

Il met la charrette dans la cour avec le chariot et les bœufs et il vient.

- Eeh! ma vertueuse femme est devenue muette, le mauvais œil est venu sur elle? Qu'est-ce que tu as fait?

- Qu'est-ce que j'ai fait? Ben, tu es venu sur moi ce matin, et puis ci et puis ça.

- Oh, mais non! Je ne suis pas venu, femme! Oh, dit-il, c'est le diable qui est venu.

Le diable est venu sur la femme du pope !

- Eh, dit-elle, tu ne vois pas que ce que dit la bouche, les fesses le disent aussi?

- Mais comment?

- Ben, est-ce que je sais, moi !

- Reste à la maison, ne sors plus, reste à la maison.

Au bout de trois jours environ, le pope... comme tout pope alla avec sa femme. Il alla avec sa femme et quand il se leva le matin, il fit sa toilette pour aller à l'église. Il prit un livre et commença à lire, il s'effraya tout seul et laissa le livre. Il commença à lire :

- Seigneur, Seigneur, Seigneur, Seigneur ! Lui :

- Seigneur, Seigneur ! Encore plus fort.

- Qu'est-ce que c'est? Ma femme ? Il dit :

- Femme! Chez toi ça parle encore?

- Non, Père, ça ne parle plus.

- Eeh ! C'est une maladie avec un remède ça!

[Le pope pour ne pas se faire moquer de lui fait dire par sa femme qu'il est malade et ne sort pas de chez lui jusqu'au soir.]

Le soir, le pope se leva et alla à l'écurie voir la jument. Il avait une jument blanche, pour partager avec elle. Pendant ce temps, il a mis... il a fait la chose... dé... déplaisante... avec la blanche et il la lâcha parmi la troupe de chevaux dans les champs.

[La jument effrayée par le bruit de sa croupe lorsqu'elle hennit courut jusqu'à mourir d'épuisement.]

Et ainsi la «maladie avec remède » fut évacuée du village grâce à l'action «... dé... déplaisante » du pope, nous y reviendrons.

Si dans le texte précédent les règles en usage concernant le rituel du mariage sont escamotées, dans celui-ci au contraire, elles sont strictement observées, y compris 
après la cérémonie. Le narrateur insiste sur le respect dû au parrain et sur le soin mis dans la préparation du cadeau à lui apporter. Selon la coutume, le parrain qui préside au baptême d'un enfant est également requis lors de la cérémonie du mariage, d'où la phrase " après que son parrain l'ait marié ». Nous avons vu plus haut que la démarche de demande en mariage (peŢit), se fait avec le parrain et/ou le père du garçon. C'est le parrain qui couvre la tête des mariés signifiant ainsi leur passage définitif dans la classe des adultes. L'hommage qui doit être rendu au parrain et à son épouse lors du toast porté en leur honneur, sărut mîna "je vous baise les mains ", atteste également de ce respect. Selon la coutume, il est entendu que le parrain et son épouse sont considérés comme des deuxièmes parents. Toutefois, dans ce texte, pas au point de leur révéler " la maladie » dont est soudainement frappée la jeune mariée.

Cette maladie s'avère avoir un remède auquel aucun des protagonistes masculins n'avait a priori pensé, l'isolement semblant être la seule réponse à cette « infirmité », et ce, toujours dans le but de ne pas «faire rire de soi ». Notons que cette maladie honteuse entre dans le foyer par l'intermédiaire des femmes et qu'elle en sort par l'intervention des hommes. Elle pourrait être vue comme une maladie sexuellement transmissible inversée puisque c'est la relation sexuelle qui, à la fois, l'amène et la guérit. La jeune femme, inexpérimentée là encore, absorbe inconsidérément une graine (une semence ?) inconnue. Soulignons encore l'éloignement du mari. Il avance sans se soucier de sa femme, qui reste seule dans un espace extérieur au village, perçu comme inconnu et dangereux (le bois, la forêt, dans d'autres récits, s'avèrent être des espaces privilégiés pour des épreuves initiatiques). De même que la jeune mariée abusée par le pope est laissée seule par son mari, celle-ci est livrée à elle-même par un mari qui s'éloigne. Aussi pourrait-on en déduire qu'il est dangereux pour les hommes de laisser leurs femmes seules. Ils sont supposés les protéger non seulement de leur naïveté (de leur bêtise ?), mais aussi de leurs instincts incontrôlés, qui finalement leur seront directement nuisibles ainsi qu'à leur foyer. Le danger pour le foyer et le village réside à l'extérieur et le vecteur en est la femme, sa bouche ${ }^{27}$ ou son sexe. On en voit pour preuve la réaction des parents de la jeune mariée qui lui demandent « ce qu'elle a fait ", comme si l'ingestion de ce petit pois représentait un adultère symbolique dont elle se serait rendue coupable. Et l'épouse du pope, abusée comme dans un des récits ci-dessus, consomme un adultère involontaire, qui à son tour sera vu comme un artifice diabolique amenant le « mauvais œil » dans le couple sacerdotal.

41 Notons aussi que le pope et sa femme sont des notables de la communauté villageoise. La femme du pope se dit preoteasă qui signifie «femme du prêtre et anciennement prêtresse », féminin de preot "prêtre ». S'il faut éviter de "faire rire de soi », ce principe vaut en premier lieu pour eux. C'est pourquoi la femme du pope dès qu'elle s'aperçoit des effets du «mauvais œil » décide d'elle-même de ni parler ni sortir. Le pope ne met pas en doute la vertu de son épouse : «ma vertueuse femme » preoteasa mea cinstită ${ }^{28}$, la confusion vient du diable et du mauvais œil. De la malveillance, en fait, et du recours à la magie, qui circule dans la communauté villageoise et dont on se garde par de nombreuses protections verbales et gestuelles.

Toute situation paradoxale ou considérée comme anormale se voit passer au crible

(...) pour déterminer à quel moment l'agression [magique] s'est manifestée.

(Andreesco, 1993, 81)

Cependant, on peut s'étonner que le pope et sa femme, s'ils sont les points convergents des regards (et de la satire des contes facétieux), puissent être les cibles d'attaques magiques, le statut de religieux faisant office de rempart. Or, la maladie arrive dans le 
foyer du pope pour atteindre le paroxysme du comique lorsque son derrière répète en écho les paroles saintes (les rires de l'auditoire et ceux du narrateur en attestent), mais aussi parce qu'il est le seul à pouvoir l'en faire sortir, non seulement de son foyer, mais aussi du village. En faisant transgresser au pope l'interdit de bestialité, le récit lui attribue, et à lui seul, un pouvoir d'exorcisme qui est (sous d'autres formes, certes) de son ressort. Le jeune marié en se délestant chez la femme du pope de sa "maladie » amorce le processus d'évacuation du mal, car malgré le mauvais tour qu'il lui joue, et dont on s'amuse, et malgré la souillure au premier degré que le pope prend sur lui, le garçon sait implicitement qu'il est le seul à pouvoir l'exorciser. Au-delà de la caricature habituelle de ces récits, où le pope est joyeusement mis en scène comme personnage libertin, il ne semble pas fortuit que cette notion de «mauvais œil » et de « diable venu sur la femme du pope » prenne cette résolution toute particulière.

\section{Les évitements de Gheorghe}

Si la scène de prières répétées par le postérieur du pope est manifestement la plus comique du récit, la conclusion du conte ne fait rire en aucune manière. Notons la gêne $\mathrm{du}$ conteur à son énonciation. Autant il se plaisait à décrire la scène de prières récitées en écho, en les répétant joyeusement, se retournant d'un air étonné, faisant des gestes et des mimiques, autant pour parler de l'accouplement pope/jument, il hésite, laisse des silences, suspend sa phrase puis dit très vite « la chose... dé... déplaisante... avec la blanche ", qu'il ne désigne plus, là, spécifiquement en tant que jument, peut-être aussi pour atténuer la portée de l'acte, qu'il ne nomme pas non plus.

Notons encore que si Gheorghe parle des organes des mariés affectés de gigantisme dans les termes les plus crus et sans aucune gêne, en revanche il désigne toujours pudiquement les relations sexuelles et n'utilise jamais le terme a regula «baiser » que Ion utilisa sans euphémisme. Dans le premier conte, le garçon «... alla avec la fille. Il coucha avec la fille ». Et le sperme par lequel le pope est rejeté est désigné «... saleté». Dans le deuxième conte, on trouve les termes : «aller avec, il lui vint l'envie, se mêler à ». L'accent n'est pas mis sur l'action sexuelle en elle-même, mais sur les maris et leurs instincts légitimes: « comme un jeune » et surtout « comme un pope »! Le pope, bien que chargé d'une fonction sacerdotale est considéré comme un mari comme les autres, et, dans le conte facétieux, comme un homme plus libertin que d'autres puisqu'il use de son statut privilégié pour séduire les femmes mariées. Aussi le conte facétieux s'emploie-t-il à le caricaturer et à l'amoindrir par les fonctions sexuelles et excrétrices, à la fois dans son état de notable du village et de prêtre; d'une part lorsqu'il transgresse les lois morales qu'il est censé représenter, d'autre part lorsqu'il est investi du pouvoir de son ministère et nimbé de son aura.

Et pour ce faire, la femme intervient à travers ces deux fonctions.

\section{La femme, entre sexe et miction}

Il est aussi intéressant de noter que la miction, dans ces récits, est exclusivement féminine, et qu'elle est également liée à la sexualité. Le pope d'un côté est rejeté par l'urine de la femme après les relations conjugales et de l'autre, la «maladie » entre dans le couple avec les conséquences que l'on sait alors que la jeune mariée est en train 
d'uriner. Dans les énoncés des narrateurs, l'expression «comme une femme ", liée à l'action d'uriner revient souvent; dans le conte du petit pois : « comme sont les femmes d'habitude "; dans celui des sexes géants : «la puce qui pique » déclenche une envie d'uriner chez la fille alors que l'attaque du loup ne déclenche aucune réaction de ce genre chez le garçon. Un autre conte faisant partie du Cycle de Păcală (voir note 11), met également en scène la miction féminine comme stratagème pour permettre au héros déguisé en mariée de se soustraire à la nuit de noces. «... tu m'attends, car moi comme une femme il faut que je pisse » (Lebarbier, 2011, 81). Le héros vide un tonneau pour contrefaire le bruit d'un liquide qui s'écoule. Et Gheorghe-Avrinte (de Breb) insiste par la répétition "et ça pissait, pissait, pissait, pissait, pissait», pour signifier l'importance du phénomène. Dans une autre variante de ce texte, racontée par MîŢa (habitante de Breb également, au parler exceptionnellement libre ${ }^{29}$ ), le marié s'étonne toutefois de la longueur de l'action: «Elle pisse beaucoup ». Le contenu d'une vessie féminine est mis en parallèle avec le contenu d'un tonneau. Soulignons que, dans aucun des textes recueillis, la miction masculine n'est mentionnée, caricaturée ou non, utilisée comme stratagème ou non.

Par ailleurs, dans la variante donnée par Gheorghe du premier conte du Cycle, Păcală, considéré comme le benêt de sa fratrie, urine et défèque sur des voleurs du haut de l'arbre sur lequel il avait trouvé refuge avec ses frères. Les voleurs effrayés s'enfuient et les garçons récupèrent leur butin. Dans cette variante-là, le héros aux caractéristiques marginales reçoit les bienfaits de son incontinence. Le sot ne peut pas contrôler ses sphincters, la femme non plus, mais la portée des récits n'est pas la même. Lorsque ce héros aux multiples facettes est lié à des motifs excrétoires, tantôt il tire son épingle du jeu et en reçoit de nombreux bienfaits; tantôt il met à mal les gobe-mouches de la noce pour s'amuser à leurs dépens. Lorsqu'il s'agit de la femme, le motif est lié soit à la sexualité et à la souillure, soit à une maladie honteuse, sexuellement transmissible, ce qui revient au même.

\section{Le discours des narrateurs}

La parole du conte facétieux apparaît plus permissive que celle de la vie courante. On ne parle pas de façon aussi crue dans la vie courante que dans le conte. L'espace du conte est un espace de liberté relative où l'on peut, sous couvert de l'histoire à raconter et à travers divers personnages, exprimer certaines situations refusées par la bienséance. Ainsi, Ioana s'excuse de sa chanson en rien "gênante ", mais cependant vécue comme telle par elle. Était-ce dû à ma présence étrangère ? À la situation de contage hors contexte ? C'était au lendemain de la veillée où ce texte avait été dit dans les rires et sans gêne apparente alors. Ou bien était-ce la situation décrite par le conte qui renvoyait à un comportement transgressif qui, plus que le chant, provoquait sa gêne ? Paulina, une autre narratrice également de Vădăstrița, s'adressa aux enfants qui m'accompagnaient dans mes enquêtes pour qu'ils ne colportent pas dans le village qu'elle s'était approprié (hors contexte elle aussi) les « bêtises » du conte facétieux. Elle se cachait derrière ma demande : «Elle m'a demandé pour rire, je lui dis pour rire ». Et ce rire-là lui semblait gênant. Comme l'est le chant de défi de la femme infidèle que Ioana reproduisit avec précautions. Ces deux narratrices redoutaient-elles les moqueries des enfants ou la désapprobation de leurs parents pour cette parole dite en 
dehors des veillées, de ces lieux de contage où la situation d'énonciation permet d'aborder des sujets tabous dans un contexte permissif.

Car on peut se demander jusqu'où peut exister la complicité entre l'émetteur et les récepteurs? Comment se situe un narrateur, plus encore une narratrice, lorsqu'il (elle) prend en charge cette parole, même en contexte. Lorsqu'il (elle) la véhicule, dans le cadre particulier d'une veillée où la parole est plus libre, et où les codes comportementaux sont plus souples, moins stricts surtout si l'on est entre personnes du même sexe, de même classe d'âge: les femmes en veillées riaient de contes et devinettes à contenu sexuel, elles étaient entre elles, « entre filles » comme on me l'a précisé. Cependant aucun mot cru ne fut proféré. Et le lendemain, devant le magnétophone, la retenue fut plus encore de mise. Qu'il s'agisse de Ioana dont nous avons vu la chanson, ou de Paulina qui conta l'histoire d'une religieuse vénale qui, malgré la tentation, "resta religieuse ». Ou encore à Breb, d'Ana contant l'histoire d'une fille pauvre qui affirmait avoir deux vagins pour se faire épouser d'un garçon riche; chacun fut doté d'un prénom, mais jamais le terme usé sans détour par Gheorghe et dénommé « le ventre » par Ion, ne fut prononcé par Ana.

Quant à Gheorghe, alors qu'il ne montre aucune gêne à nommer les organes génitaux dans leurs termes les plus crus, nous le voyons user d'euphémismes pour parler de la miction de la jeune mariée, de la relation bestiale du pope et de la jument, ou des relations conjugales. En revanche, Ion nomme les relations sexuelles dans un des termes les plus populaires. Petru, l'adolescent, lui, va "l'apprendre », mais demande « une bite » à son père! Alors on peut en déduire que le seuil de ce qui est dicible et de ce qui ne l'est pas est variable pour chacun, chacune ; variables les mots qui souillent et ne peuvent être dits et ceux qui peuvent être prononcés sans dommage; variables les actions qui peuvent être décrites, et celles qui ne peuvent être qu'évoquées.

51 À l'examen de ce corpus, on peut simplement en conclure que si les narratrices prennent plus difficilement en charge leurs énoncés et restent dans l'allusion, les narrateurs établissent une distance qui diffère selon l'action ou l'objet abordés et selon, probablement, leur personnalité et leur situation dans le village.

Quant à assumer ses dires devant un auditoire, composé de familiers, il est clair que cette parole-là expose. Mais on ne s'expose pas n'importe comment, devant n'importe qui. On le fait devant ses pairs, pas devant une autre classe d'âge, ou devant un étranger. Et devant l'étrangère venue recueillir ces récits "pour la science $"^{30}$, on ne peut aborder ces thèmes qu'avec précautions, détours ou hors de sa présence ${ }^{31}$. Car le regard de la communauté ne doit jamais être moqueur pour soi-même, "ne pas faire rire de soi». On peut s'accommoder en revanche de faire rire aux dépens de personnages dont on veut amoindrir le poids moral (le pope) ou fantasmé (la femme), par l'intermédiaire de la caricature du conte facétieux. L'image caricaturée et les dires outranciers qui l'accompagnent seraient proportionnels au poids dont on souhaiterait s'alléger. 


\section{BIBLIOGRAPHIE}

ANDREESCO, Ioana, 1993, Magie et destin ou de l'inconvénient de vivre à deux (Roumanie), Cahiers de littérature orale, $\mathrm{n}^{\circ} 34, \mathrm{p} .77-93$.

AARNE, Anti, THOMPSON, Stith, 1964 (2nd ed.), The types of Folktales. A classification and bibliography, Anti Aarne's Verzeichnis der Märchentypen, Translated and enlarged by S. Thompson, Indiana University, second revision, Helsinki, Academia Scientiarum Fennica, F.F. Communications No 184.

DEX, Dicţionarul explicativ al limbii române [Dictionnaire explicatif de la langue roumaine], 1996, București, Univers enciclopedic (ediŢia a IIa).

LASCAULT, Gilbert, 2008, Figurées défigurées. Petit vocabulaire de la féminité représentée [article « Dévoration »], Paris, Éditions du Félin [1e éd. 1977].

LEBARBIER, Micheline, 1976, Les notables libertins, Cahiers de littérature orale, $\mathrm{n}^{\circ} 1$, Paris, Publications orientalistes de France, p. 135-156.

LEBARBIER, Micheline, 1987, Identité culturelle et littérature orale, Variations autour d'un personnage facétieux roumain, Actes du Colloque Kalevala et traditions orales du monde, in M. M. J. Fernandez-Vest (éd.), Paris, Éditions du CNRS, p. 411-422.

LEBARBIER, Micheline, 1989, L'identité dénaturée, Păcală et le Tsigane dans les contes facétieux roumains, in F. de sIVERS (éd.), Questions d'identité, Louvain-Paris, Peeters-SELAF, p. 133-150.

LEBARBIER, Micheline, 1998, L'ordre et le culte : comique et rire dans les contes facétieux roumains, in Éliane DAPHY et Diana REY-HULMAN (dir.), avec la collaboration de Micheline LEBARBIER, Paroles à rire, Paris, coll. « Colloques Langues'O », INALCO, p. 165-179.

LEBARBIER, Micheline, 2000, Les dires du conte, l'exemple de quelques contes facétieux roumains, in Bertrand MASQUELIER et Jean-Louis SIRAN (dir.), Pour une anthropologie de l'interlocution. Rhétoriques du quotidien, Paris, L'Harmattan, p. 247-280.

LEBARBIER, Micheline, 2009, « Păcală », ou la fortune de l'absurde, Cahiers de littérature orale, $\mathrm{n}^{\circ} 66$ « Mémoire des CLO », Paris, Publications Langues O', p. 147-163 [1 ${ }^{\text {re }}$ éd. 1976].

LEBARBIER, Micheline, 2011, Contes à rire de Roumanie. Facéties et histoires courtes, Paris, Karthala Langues O', coll. « Paroles en miroir », $\mathrm{n}^{\circ}$ 6, $284 \mathrm{p}$.

LÉVI-STRAUSS, Claude, 1962, La pensée sauvage, Paris, Plon, 393 p.

LÉVI-STRAuss, Claude, 1964, Mythologiques. Le cru et le cuit, Paris, Plon, 402 p.

LÉVI-STRAUSS, Claude, 1966, Mythologiques. Du miel aux cendres, Paris, Plon, 450 p.

MARIAN, S. Fl., 1985, Naşterea la Români, studiu etnografic [La naissance chez les Roumains, étude ethnographique], Bucureşti, Editura grai şi suflet - Cultura naŢională, 277 p. [1 $1^{\text {re }}$ éd. 1892].

STROESCU, Sabina Cornelia, 1969, La typologie bibliographique des facéties roumaines, Bucarest, Éditions de l'Académie de la République socialiste de Roumanie, 2 vol., 1767 p.

YACINE, Tassadit, 2011, L'art de dire sans dire en Kabylie, Cahiers de littérature orale, $\mathrm{n}^{\circ} 70$, « L'adresse indirecte », Paris, Publications Langues O', p. 67-86. 


\section{NOTES}

1. Une correction corporelle, même violente, est reconnue comme méritée voire légitime si elle émane du mari trompé qui démasque l'infidèle et son amant, ou s'il veut corriger son épouse paresseuse et lui donner le goût des tâches domestiques. Mais parfois, la honte publique apparaît comme le châtiment le plus efficace pour remettre l'épouse récalcitrante dans le droit chemin. La plupart des personnes interrogées étaient soucieuses de «ne pas faire rire (d'elles) dans le village ».

2. Je reprends ici la terminologie utilisée par S.C. Stroescu dans son imposante Typologie bibliographique des facéties roumaines $(1969, \mathrm{xI}):$ « (...) Le motif représente une réunion d'éléments dans une relation qui lui donne un certain contenu déterminé par la mentalité de la collectivité créatrice (...) L'élément représente la plus petite partie constitutive d'une narration. »

3. Les motifs scatologiques sont le plus souvent corrélés aux motifs sexuels, notamment lorsqu'il s'agit de la miction féminine. Aussi je traiterai principalement de ceux-ci dans le cadre de cet article. Je ne m'attarderai pas sur d'autres motifs scatologiques (notamment ceux qui concernent un Tsigane sot et malchanceux, ou ceux, métaphoriques, qui souillent les notables punis d'avoir convoité une femme mariée).

4. Voir Lebarbier (2000, 263 et ss) " "Autrement dire": les équivalents symboliques de l'adultère ».

5. Pour plus de détails sur ces récits et leur étude, voir le chapitre consacré aux travers féminins dans Lebarbier (2011, 99-157). Dans le cadre de cet article, je me bornerai à examiner les descriptions explicites ou métaphoriques données par les narrateurs sur ces motifs et d'en dégager autant que possible un sens.

6. Recueillie auprès de Dumitru, habitant d'un village proche de Sibiu. Il était venu à l'Institut de Folklore de Bucarest offrir son répertoire de récits aux chercheurs de l'institut. J'ai pu assister à cette séance de contage (hors contexte là aussi) et eu l'autorisation de l'enregistrer.

7. Alors que dans la première variante, dite par Dumitru, il est demandé à la femme de « servir » le soi-disant « marchand de prunes ».

8. Bien qu'il doive observer la scène de loin, « de la porte », il paie ce « service » cinq cents lei, somme importante alors. Doublement berné, le mari !

9. Les références les plus évidentes à ce mythe renvoient bien sûr à Lévi-Strauss $(1962,141$; $1964,121 ; 1966,171,200,264)$.

10. Bien qu'en âge de se marier, le protagoniste ignore tout de l'anatomie féminine, ses tentatives d'apprentissage ayant échoué, nous l'avons vu. Mais il est également ignorant de sa propre anatomie, du moins de sa désignation, puisqu'il demande à son père « de lui donner une bite » le jour de ses noces...

11. On retrouve ce héros dans de nombreux contes facétieux, un Cycle de récits lui est consacré. Pour plus de détails sur ses multiples caractéristiques voir Lebarbier (1987, 1989, 2009, 2011, 21-98). Ailleurs il s'appelle Nastratin Hodgea ou encore Till l'Espiègle (Lebarbier, 2011, 23, note 6).

12. Le pope, libertin souvent, et amateur de femmes mariées, peut aussi être un mari trompé.

13. Mes commentaires sont entre crochets, la phrase entre parenthèses est dite en aparté par la narratrice.

14. « ... selon la coutume, une femme enceinte, lorsqu'elle voyait que son terme approchait, devait aller à l'église pour se confesser et communier. » (Marian, 1995, 31), je traduis.

15. Je traduis.

16. $C f$. note 12. Le pope est fréquemment l'instigateur d'une transgression ou une victime impuissante et ridicule dès lors qu'un motif sexuel apparaît dans un conte : coureur invétéré et diversement châtié par le mari, mari trompé, ou encore mari berné (ou les deux comme nous le voyons ici). 
17. Certes, le pope profite de la naïveté de la jeune femme, mais il abuse de son pouvoir religieux ainsi que de sa posture de confesseur face au confessé. Dans le rite orthodoxe, le pope est debout et le pénitent à genoux devant lui. Il lui recouvre le dos de son étole (patrafir), ce qui induit une forme de promiscuité physique dont le pope profite dans ce conte... et qui peut-être est redoutée par la population.

18. Ou d'autres types de châtiment qui ont été examinés ailleurs (Lebarbier, 1976; 2011, 214-229). Soit il doit se cacher, lorsque le mari arrive, dans un tonneau plein de lie-de-vin et être pris pour un diable lorsqu'il s'en échappe, soit il doit payer le silence du mari ou encore lui moudre jusqu'au matin (et au moulin à bras) six mesures de grains de maïs.

19. Manier ces termes dans une autre langue est plus aisé que dans la sienne. Dire dans sa langue confronte aux tabous de sa propre société et aux siens propres ( $c f$. Yacine, 2011, 78 et ss « La langue de l'autre: un détour pour se dire»). Dire dans une autre langue permet de "se situer dans un autre monde, prendre de la distance y compris par rapport à soi-même à "sa culture, son appartenance à un groupe déterminé, sa sexualité”... » (ibid., 82).

20. C'est ce texte qui ne put être enregistré qu'en présence d'un auditoire exclusivement masculin (cf. $\S$ « La place de ces motifs dans le corpus »).

21. Taica: terme familier et affectueux pour tata, "papa ", que l'on peut traduire par "petit père, petit papa » et qui peut être utilisé pour marquer l'affection en dehors des relations de parenté. Le terme tata sera utilisé un peu plus loin lorsque le futur gendre interpelle son futur beau-père.

22. C'est ici que le narrateur dit tata « papa ».

23. Le narrateur mime quelqu'un qui court et qui est essoufflé.

24. Une ballade entendue lors d'une soirée familiale : Blestemat de mamă « Maudit par sa mère », décrit avec force détails hyperboliques les tribulations d'un garçon que sa mère avait chassé et maudit.

25. Je n'ai recueilli que deux textes où il était question de malformation physique (il s'agissait de bossus en l'occurrence) car m'a-t-on affirmé : «On ne rit pas des défauts physiques, ce serait un péché ", mais on rit du gigantisme des sexes... masculins, qui alors ne seraient pas vus comme une infirmité, mais servirait l'hyperbole sur laquelle repose le burlesque de la narration!

26. La quarantaine que devait respecter la femme récemment accouchée confirme cette notion de souillure (Marian, 1995, 87).

27. La bouche des femmes... est fréquemment mise à mal dans les contes facétieux. Elles mentent, invectivent leurs maris, vont colporter leurs secrets qui se trouvent déformés et arrivent à l'oreille de l'empereur "de bouche en bouche de femmes" précise le narrateur (Lebarbier, 2000, 279). Ici le maléfice que représente « la bouche des femmes » se trouve intriqué entre verbal, oral et sexuel.

28. La vertu des femmes est parfois mise en relief dans les récits de Gheorghe. Dans un ensemble de textes (répertorié chez Aarne T. 1730) où des notables libertins visitent à tour de rôle une femme mariée, la femme et son mari sont implicitement complices pour les ridiculiser ou les faire payer. Dans la variante de Gheorghe le mari est loin, met en doute la fidélité de sa femme et la tue. Or Gheorghe insiste en ces termes sur la vertu de son personnage : "Elle était honnête (cinstită), Despina, tellement honnête que ce n'est plus possible (încît nu se mai poate) ».

29. Cette narratrice n'hésitait pas à introduire des motifs scatologiques dans ses récits, alors qu'ils n'existaient pas dans les autres variantes recueillies ni dans celles répertoriées dans la Typologie de S.C. Stroescu. Par exemple, dans l'épisode suivant de ce même récit raconté par MîŢa, Păcală fait boire un purgatif aux participants de la noce, qui se retrouvent souillés et fort gênés le lendemain matin.

30. Argument employé par les chercheurs de l'Institut d'Ethnographie et de Folklore pour délier la parole des interlocuteurs.

31. Rappelons que le récit des sexes géants fut enregistré hors de ma présence. 


\section{RÉSUMÉS}

À travers l'étude de plusieurs contes facétieux recueillis dans trois villages de la Roumanie communiste, les motifs qui ont trait à la sexualité et à la miction féminines sont répertoriés. La récurrence de ces motifs, l'implication ou la distance des narrateurs vis-à-vis de leurs énoncés sont examinées en fonction du contexte de l'énonciation, de leur classe d'âge, de leur genre, ainsi que les mots pour exprimer ou pour suggérer.

Based on the study of several facetious tales collected in three Rumanian communist villages, we have established the repertoire of motifs pertaining to sexuality and female micturition. The recurrence of these motifs, the implication of the narrators or their distancing with respect to what they say is examined within the context of performance, age grade, and gender. The words used to express or suggest these motifs are also studied.

\section{INDEX}

Mots-clés : contes facétieux, motifs sexuels, miction féminine, narration Index géographique : Roumanie

Thèmes : anthropologie (Europe)

Keywords : Facetious Tales, Rumania, Sexual Motifs, Female Micturition, Male Narrators, Female Narrators, Discourse, Context 